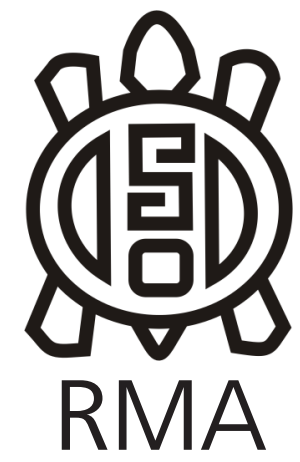

Dossier

\title{
Artefactos picados, abradidos y/o modificados por uso en Norpatagonia. El caso del sitio Loma de los Muertos (pcia. de Rio Negro)
}

Chopped, abraded and/ or modified by use artifacts in Norpatagonia. The case of the site Loma de los Muertos (Rio Negro province)

Daniela Saghessi*

*CONICET, División de Arqueología, Facultad de Ciencias Naturales y Museo, Universidad Nacional de La Plata, Argentina. E-mail: danisaghessi7@gmail.com

\begin{abstract}
Resumen
En este trabajo se presentan los resultados del análisis tecno-morfológico y morfológico-funcional de los artefactos líticos picados, abradidos y/o modificados por uso procedentes del sitio arqueológico Loma de los Muertos (LM) (dpto. de General Conesa, pcia. de Rio Negro). El sitio, ubicado en la margen sur del valle del río Negro, fue interpretado como un campamento residencial de actividades múltiples en el que además se realizaron entierros humanos, en al menos cuatro episodios diferentes de ocupación a lo largo del Holoceno tardío. Hasta el momento, sólo se cuenta con un análisis general de los artefactos picados y/o abradidos del sitio. Esta situación concuerda con lo que sucede en otros sectores de Patagonia donde estos instrumentos no han constituido hasta ahora fuentes de información arqueológica acordes a su potencial. Los objetivos principales de este trabajo son determinar las materias primas utilizadas e identificar las fuentes de aprovisionamiento, y reconocer los procesos principales de producción y uso de cada grupo tipológico. A través de ello se busca estimar los rangos de movilidad implicados en la obtención de las rocas e inferir las actividades desarrolladas en el sitio vinculadas con los artefactos picados, abradidos y/o modificados por uso.
\end{abstract}

Palabras clave: Norpatagonia; artefactos picados, abradidos y/o modificados por uso; análisis tecno-morfológico; análisis morfológico-funcional; Loma de los Muertos.

\begin{abstract}
This paper presents the results of the techno-morphological and morphological-functional analysis of the lithic artifacts made by chopped, abrasion and/or modified by use, from the archaeologic site of Loma de los Muertos (LM) (Northern Patagonia, Argentina). The site is located in Río Negro valley and was interpreted as a residential camp where hunter-gatherer groups carried out domestic and inhumation activities in at least four different occupation episodes during the late Holocene. Until now, a general description of these ground stones is available. This situation is similar to what happens in other areas in Patagonia, where these artifacts were not studied systematically. The main aims of this paper are to determine the raw materials used and to identify potential quarry deposits/outcrops; and to recognize the main processes of production and use of typological groups. The purpose of this study is to infer mobility ranges involved in obtaining the rocks, and to infer the activities developed on the site related to the artifacts production by chopped, abrasion and/or modified by use.
\end{abstract}

Keywords: Norpatagonia; ground stones; techno-morphological analysis; morpho-functional analysis; Loma de los Muertos.

Los artefactos picados, abradidos y/o modificados por uso han recibido una atención creciente durante los últimos años en estudios de cazadores recolectores. A través de varias vías de estudio (por ej. arqueobotánicos, petrológicos y experimentales) han aportado información diversa sobre la vida de las sociedades cazadoras recolectoras y, en especial, sobre su subsistencia (Álvarez Soncini, 2018; Babot, 2011; Lema et al., 2012; Matarrese, 2015; Vecchi, 2011; Vecchi y González, 2018; entre otros). Esto ha permitido, entre otras cosas, evaluar los cambios en los modos e intensidad de la explotación de recursos vegetales a través del tiempo (Babot, 2006;
Heider y López, 2016; Matarrese, 2015; entre otros). En este trabajo, se presentan los resultados del análisis tecno-morfológico y morfológico-funcional del conjunto de artefactos líticos picados, abradidos y/o modificados por uso procedentes del sitio Loma de los Muertos (en adelante LM) (provincia de Rio Negro), de los cuales solo se había efectuado una descripción general (Prates et al., 2010a). Los objetivos principales de este trabajo son determinar las materias primas utilizadas e identificar las fuentes de aprovisionamiento, y reconocer los procesos principales de producción y uso de cada grupo tipológico. A través de ello, se busca estimar los rangos de movilidad 
implicados en la obtención de las rocas e inferir las actividades desarrolladas en el sitio vinculadas con los artefactos picados, abradidos y/o modificados por uso.

El sitio LM se ubica en el sector sur del valle del río Negro $\left(40^{\circ} 08^{\prime} 47,03^{\prime \prime} \mathrm{S}\right.$ y $\left.64^{\circ} 16^{\prime} 19,54^{\prime \prime} \mathrm{O}\right)$ y a $1,4 \mathrm{~km}$ al SO del cauce actual del río (Figura 1). Se encuentra sobre un médano, en inmediaciones de una laguna, y fue descubierto accidentalmente mientras se realizaban la remoción de tierra para tareas agrícolas. Fue interpretado como una base residencial donde se llevaron a cabo actividades múltiples y se realizaron entierros humanos, en al menos cuatro episodios diferentes de ocupación a lo largo del Holoceno tardío (Mange et al., 2013; Prates et al., 2010 a y b; Prates, 2014). Dentro de las actividades que fueron asociadas con la ocupación residencial se encuentran el procesamiento y consumo de diversos recursos faunísticos (Lama guanicoe, Ozotoceros bezoarticus, Rhea americana, Pterocnemia pennata y varias especies de roedores); el uso de recipientes de cerámica para, posiblemente, la cocción de alimentos y para la explotación más eficiente de animales acuáticos (Di Prado, 2013); y la manufactura y utilización de artefactos líticos tallados (raspadores, artefactos de formatización sumaria, puntas de proyectil, núcleos y desechos de talla) y de artefactos picados, abradidos y/o modificados por uso analizados en este trabajo (véase Prates et al., 2010a).

\section{Materiales y Métodos}

El análisis de los artefactos se realizó siguiendo los lineamientos propuestos por Aschero $(1975,1983)$, Adams (2002), Babot (2004) y Matarrese (2015). La evaluación de la procedencia de las materias primas se realizó teniendo en cuenta sus atributos macroscópicos, las observaciones realizadas en el campo (Mange, 2019; Prates, 2008) y las descripciones de varias formaciones geológicas (Andreis, 1965; De Ferrariis, 1966; González Díaz y Malagnino, 1984; Bonomo y Prates, 2014). Para el estudio tecno- morfológico y morfológico-funcional se consideraron variables de estado (alteraciones naturales y estado de conservación de la pieza), dimensionales (dimensiones absolutas), técnico-morfológicas (materia prima, tamaños de granos, modalidad de manufactura), y morfológicofuncionales (tipo de artefacto según el número y función de las zonas activas, rastros de uso macroscópicos, grupo tipológico, designación morfológico-funcional del artefacto). El instrumental utilizado fue calibre metálico y cinta métrica para las variables dimensionales, y lupa de mano (10X) para las variables técnico-morfológicas y las morfológico-funcionales.

\section{Resultados}

En el conjunto analizado $(n=40)$ se identificaron los siguientes grupos tipológicos: instrumentos de molienda $(n=25)$, bolas de boleadora $(n=9)$, instrumentos compuestos $(n=3)$ y piezas indiferenciadas $(n=3)$. La mayoría de los artefactos se encuentran fragmentados $(n=23)$. El $45 \%$ de las piezas fueron confeccionadas a partir de rodados de rocas ígneas básicas (basalto), ígneas ácidas y metamórficas. Además, se utilizaron areniscas (55\%) (Tabla1). Los atributos macroscópicos más destacables de los rodados son formas facetas y chatas, y longitudes superiores a $10 \mathrm{~cm}$. De acuerdo a estas características, a las descripciones geológicas y a las observaciones realizadas en el campo (Bonomo y Prates, 2014; Mange, 2019; Prates, 2008), los rodados aquí utilizados podrían provenir de los mantos de rodados fluviales depositados por el río Negro. En el caso de las areniscas, las tonalidades griseas (claras y oscuras), el tamaño de grano medio a fino y el grado variable de friabilidad, entre otros aspectos, sugieren que esta materia prima procede de la Formación Río Negro (Andreis, 1965; De Ferrariis, 1966).

Dentro de los instrumentos de molienda, se determinaron grupos tipológicos de posición inferior (mortero $n=1$, molino $n=7$ y pasivos indefinidos $n=3$ ), de posición

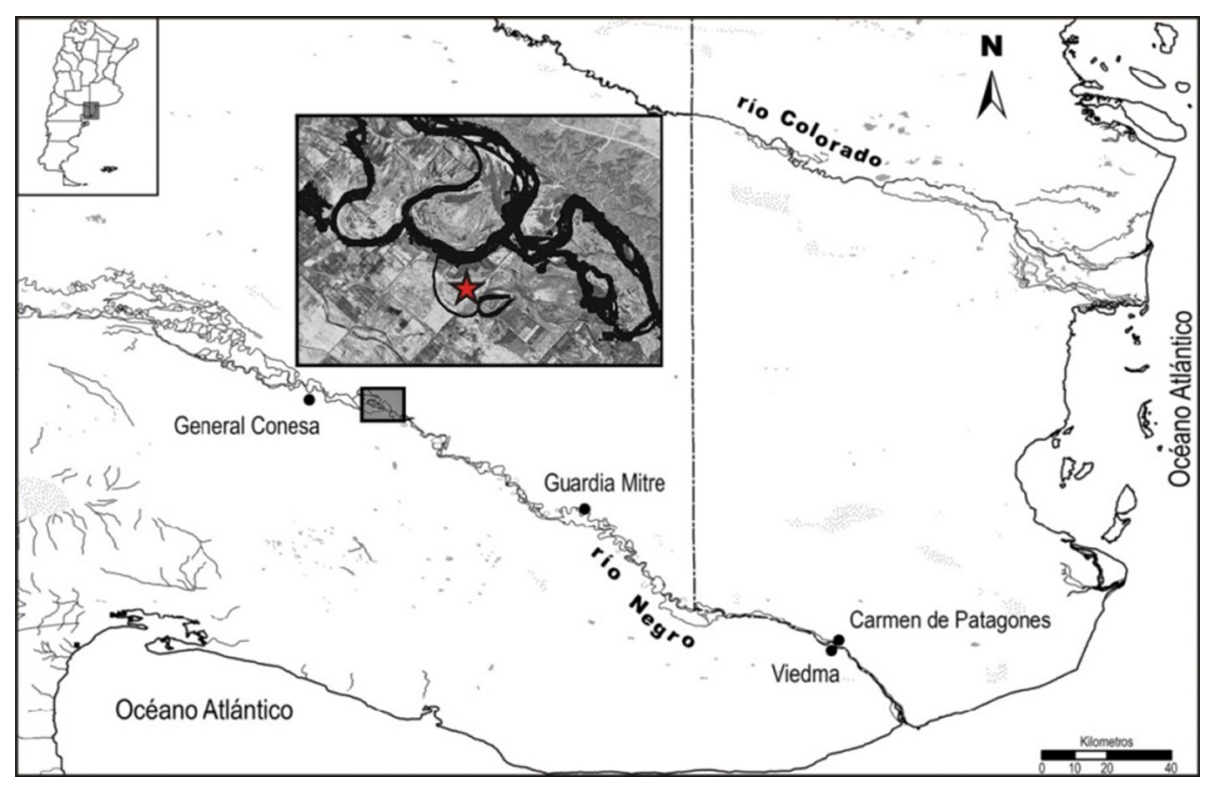

Figura 1. Mapa de la ubicación del sitio Loma de los Muertos (tomado de Prates et al., 2010a)

Figure 1. Map of the location of the site Loma de los Muertos (taken from Prates et al., 2010a) 
Tabla 1. Uso de materia prima por grupo tipológico.

Table 1. Use of raw materials by typological group.

\begin{tabular}{|c|c|c|c|c|}
\hline & \multicolumn{3}{|c|}{ Rodados } & \multirow{2}{*}{ Areniscas } \\
\cline { 2 - 5 } & $\begin{array}{c}\text { Ígneas } \\
\text { ácidas }\end{array}$ & $\begin{array}{c}\text { Ígneas } \\
\text { básicas }\end{array}$ & Metamórficas & 19 \\
\hline Inst. de molienda & 5 & 1 & - & 3 \\
\hline Bolas de boleadoras & 6 & - & - & - \\
\hline $\begin{array}{c}\text { Percutores y } \\
\text { Yunques }\end{array}$ & 3 & - & - & - \\
\hline Indiferenciadas & 1 & - & 2 & \\
\hline
\end{tabular}

superior (mano de mortero $n=1$, mano de molino $n=2$, activos compuestos $n=5$, activos indefinidos $n=2$ ) y de posición indeterminada $(\mathrm{n}=4)$. La mayoría, tanto pasivos (mortero y molino) como activos (mano de mortero y mano de molino), son simples $(n=11)$, es decir que tienen una sola oquedad o superficie activa (Babot, 2004). En menor medida se registraron instrumentos compuestos solo de posición activa (mano de molino/sobador y mano de mortero/abradidor) ( $n=5)$. En el $56 \%$ de los instrumentos se emplearon una o más técnicas de manufactura en una o varias caras, siendo pocos los casos en los que la pieza entera presentaban formatización (12\%). Aquí se incluyen la totalidad de los artefactos pasivos antes mencionados y la mayoría de los activos. El 44\% restante solo muestra evidencias de modificación por uso, posiblemente debido a la alta fragmentación del conjunto. Este fue el caso de los artefactos de posición indeterminada y de los activos indefinidos, los cuales están representados solo por un fragmento de superficie activa. Los procedimientos tecnológicos aplicados durante la manufactura fueron el picado para regularizar contornos, caras de apoyo, caras activas y zonas de prensión; y la abrasión (alisado, pulido y/o bruñido) para dar acabado a las superficies. Los rastros de uso macroscópicos más frecuentes en las superficies activas de artefactos activos y pasivos son las estrías oblicuas y paralelas, los microlascados y el alisado (Semenov, 1964 citado en Babot, 2004). Además, en dos piezas se registraron restos de sustancias adheridas. La primera es un molino de basalto con una coloración rojiza en la oquedad, posiblemente pigmento (Figura 2A); y, la segunda, un mortero de arenisca gris que, en su superficie activa, se identificó una adherencia de tonalidad oscura, posiblemente material vegetal carbonizado (Figura 2B). Respecto a las dimensiones relativas y a la forma general de las piezas, en los artefactos pasivos predominan las categorías "espesos" 1 y "cortos", con formas generales discoidales; y en los artefactos activos las categorías de "muy espesos" y "medianos", con una diversidad mayor de formas generales (discoidal, ecuante, laminar, esférica) (sensu Babot, 2004). Se registraron tres casos de reciclaje de artefactos pasivos y, en cuanto a la condición de descarte, la mayoría de las piezas están agotadas.

Dentro de las bolas de boleadora $(n=9)$, se reconocieron

\footnotetext{
"Siguiendo lo planteado por Babot (2004), las categorías "mediano", "corto", "muy espeso" y "espeso" son algunas variantes de los módulos de longitud y de espesor, los cuales corresponden a la razón entre dimensiones máximas de una pieza (largo, ancho y espesor).
}

piezas enteras $(n=4)$ y fracturadas $(n=5)$. Son todas lisas, de tamaño variable (entre 3,4 y 7,5 cm de diámetro) y ninguna presenta surco perimetral. Fueron manufacturadas por picado y abrasión. En el caso de las dos preformas, ambas están fracturadas, son subesféricas y tienen huellas de picado grueso (sensu Babot, 2004); en una de ellas se observó corteza de rodado (Figura $3 \mathrm{H}-\mathrm{I}$ ). Con respecto a los instrumentos compuestos $(n=3)$, se registró una combinación de superficies activas de percutores y yunques (Figura 4), con categorías de "medianos" y "muy espesos" para los módulos de longitud y de espesor. Todas estas piezas fueron descartadas con remanente de vida útil y desgaste moderado-fuerte de sus superficies. Por último, hubo tres casos en los que no se puedo definir el grupo tipológico (indiferenciadas) ya que no presentan evidencias de manufactura y sus rastros de uso no son claros.

\section{Discusión}

\section{Procedencia y uso de materias primas}

Para la confección de los artefactos picados, abradidos y/o modificados por uso del sitio LM se utilizaron posiblemente solo materias primas de procedencia local. El sitio se ubica en la sección inferior de la planicie aluvial del río Negro -primera terraza de inundación (T1) o sector intermedio (sensu Prates, 2008). Esta localización en una

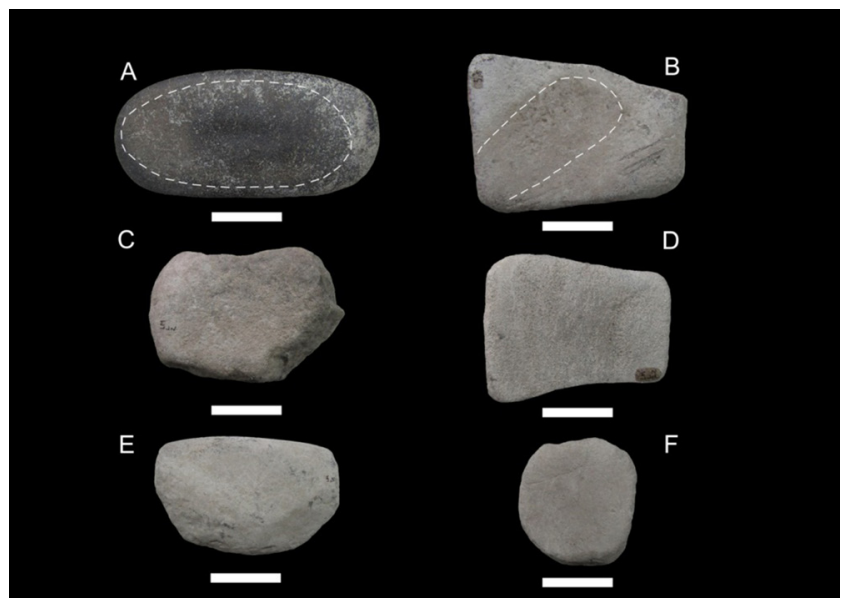

Figura 2. Instrumentos de molienda. Referencias: A, B, C y D instrumentos pasivos; $\mathrm{E}$ y $\mathrm{F}$ instrumentos activos. Escala: $5 \mathrm{~cm}$

Figure 2. Grinding tools. References: $A, B, C$ and $D$ passive artifacts; $E$ and $F$ active artifacts. Scale: $5 \mathrm{~cm}$ 


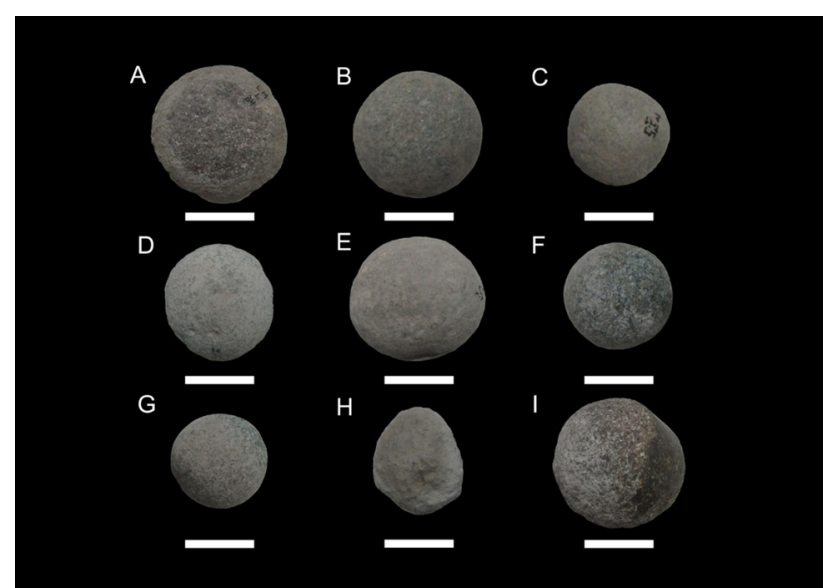

Figura 3. Artefactos compuestos (percutores y yunques). Escala: $5 \mathrm{~cm}$

Figure 3. Composite artifacts (hammers and anvils). $5 c$ ale: $5 \mathrm{~cm}$

porción del valle donde predominan sedimentos fluviales (gravas, arenas, limos y arcillas) y eólicos (arenas y limos) y se caracteriza por paleocauces, lagunas y paleolagunas (Luchsinger, 2006), habría brindado un fácil acceso a las fuentes de aprovisionamiento.

Los depósitos de rodados fluviales se encuentran disponibles casi de forma continua a lo largo de la cuenca del río Negro desde momentos post-glaciales y están compuestos por diferentes tipos de rocas (basaltos, andesitas, riolitas, granitos, dacitas, sílices) (Bonomo y Prates, 2014; Mange, 2019). En el conjunto analizado se distinguieron algunos criterios de selección dentro de la diversidad de rodados. En primer lugar, se habría optado por aquellos de dimensiones grandes (mayores a $10 \mathrm{~cm}$ de largo) y formas facetadas y chatas. Si bien estos tipos son poco frecuentes en los depósitos permite diferenciarlos de los rodados patagónicos, los cuales son más pequeños (entre 3 y $5 \mathrm{~cm}$ de diámetro) y redondeados (Bonomo y Prates, 2014), y además explica la prevalencia de la categoría "mediano" en el módulo de longitud de los instrumentos compuestos. En segundo lugar, los rodados utilizados, principalmente riolitas y basalto, se caracterizarían por un mayor grado de dureza y una menor calidad para la talla que las rocas silíceas también disponibles en los depósitos.
En los sectores altos del valle (borde externo del valle o terrazas pleistocénicas -T2 y T3- sensu Prates, 2008) se habrían obtenido las areniscas. En el área de estudio se han descripto dos sectores principales con disponibilidad de esta materia prima: el valle del río Negro y las barrancas de la costa norpatagónica. En el caso del río Negro afloran las areniscas grises como parte de la roca madre excavada por el río en sus tramos medio e inferior (Escosteguy et al., 2011; Suriano et al., 1999), a causa de la interrupción de la antigua planicie aluvial disecada del noreste patagónico (sensu González Díaz y Malagnino, 1984). Las características macroscópicas de las areniscas identificadas (e.g. color, tamaño de grano y friabilidad), coinciden con las descripciones existentes para las areniscas de la Formación Rio Negro (Andreis, 1965; De Ferrariis, 1966; González Díaz y Malagnino, 1984), las cuales son consideradas por diversos trabajos arqueológicos como un recurso lítico ampliamente utilizado por los grupos que habitaron el área (Mange, 2019; Prates, 2008).

\section{Proceso de formatización y uso}

Dentro de las diversas actividades realizadas en LM se encuentra la manufactura de los artefactos aquí analizados que se infiere, por ejemplo, del registro de preformas de bolas de boleadoras con corteza y rastros de picado grueso (sensu Babot, 2004). Además, la existencia de percutores y yunques se relaciona con los procesos de manufactura de otros instrumentos en el sitio (por ej. instrumentos tallados). Un aspecto interesante sobre la formatización de los artefactos picados, abradidos y/o modificados por uso es la relación entre las materias primas empleadas y los distintos grupos tipológicos identificados. Mientras que el uso de arenisca está vinculado mayormente con aquellos grupos tipológicos caracterizados por superficies abrasivas (artefactos de molienda), el uso de rodados está asociado a los tipos de artefactos en los que la dureza es un atributo deseable ya que necesitan soportar golpes o ser arrojados (percutores, yunques y bolas de boleadoras).

Con respeto a los artefactos de molienda, la falta de evidencias de las primeras etapas de formatización no permite asegurar que el proceso haya comenzado en el sitio. Sin embargo, el registro de tres casos de reciclaje (por ej. de molino doble a un molino simple; o de un artefacto

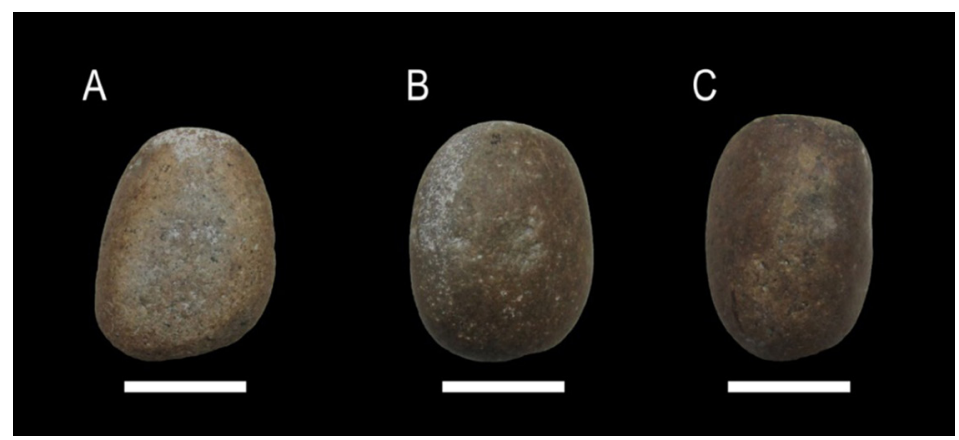

Figura 4. Bolas de boleadoras. Referencias: piezas enteras $(B, C, F, G)$, piezas fracturadas $(A, D, E)$ y preformas $(H, I)$. Escala: $5 \mathrm{~cm}$

Figure 4. Boleadora balls. References: whole pieces $(B, C, F, G)$, fractured pieces $(A, D, E)$ and preform $(H$, I). Scale: $5 \mathrm{~cm}$ 
indeterminado a un artefacto activo compuesto -mano mortero y abradidor-) y de la reactivación de la oquedad de un molino por picado permite plantear que en LM se habría dado la reformatización de algunos artefactos. El número de procedimientos tecnológicos empleados para la manufactura, y con ello la complejidad del proceso, estaría relacionado con el tipo de materia prima. En los instrumentos de arenisca se registraron, en la mayoría de los casos, series técnicas que combinaron el picado y la abrasión en alguna de sus tres fases (alisado, pulido y bruñido). En cambio, en las piezas confeccionadas sobre rodados el proceso de formatización fue nulo para la mayor parte de los artefactos, o sólo implicó el empleo de un procedimiento tecnológico, como el picado o el lascado.

Los artefactos aquí analizados habrían estado implicados en diferentes actividades vinculadas a la subsistencia, entre ellas la caza (Mange et al., 2013) y el procesamiento de recursos vegetales y de pigmentos (Casamiquela, 1999; Prates, 2009; Saghessi y Mange, 2017). Para corroborar estos supuestos es necesario realizar estudios complementarios, como arqueobotánicos y funcionales (Álvarez Soncini, 2018; Babot, 2011; Lema et al., 2012). Entre los aspectos más destacables del conjunto en relación al uso pueden mencionarse, en primer lugar, que todas las piezas son de tamaños relativamente pequeño y transportable, lo que no permite pensar en una estrategia de equipamiento del sitio (Binford, 1979). En segundo lugar, que la prevalencia de artefactos agotados (principalmente artefactos de molienda) y artefactos con remanente de vida útil pero con desgaste moderado-fuerte de sus superficies (bolas de boleadora y artefactos compuestos) sería producto de un uso intensivo. Por último, el bajo grado de formatización y de estandarización morfológica de los instrumentos de molienda podría responder a un uso expeditivo de las materias primas (Nelson, 1991).

\section{Conclusión}

El estudio de los artefactos líticos picados, abradidos y/o modificados por uso de Loma de los Muertos permitió caracterizar de modo general el conjunto del sitio. Concretamente se determinó que:

- Las materias primas utilizadas fueron locales, las fuentes de aprovisionamiento habrían estado en las cercanías del sitio. Los rangos de movilidad implicados en la obtención del material rocoso no habría llegado más allá de los sectores altos del valle donde aflora la arenisca gris de la Formación Río Negro.

- La mayor parte del proceso de formatización de los distintos artefactos picados y/o abradidos se habría realizado en el sitio. En el caso de los artefactos de molienda, la complejidad de la manufactura y el número de procesos tecnológicos implicados estaría en relación con el tipo de materia prima. Los instrumentos confeccionados en arenisca tienen series técnicas más complejas, en cuanto al número de procesos tecnológicos implicados, que los artefactos manufacturados sobre rodados.

- Los artefactos analizados habrían sido elementos útiles para los grupos que habitaron el sitio. Esto se infiere, por ejemplo, a partir del uso intensivo que recibieron las piezas en distintas actividades asociadas a la subsistencia y a la manufactura de otros instrumentos, y de la posibilidad de transportar estos instrumentos siguiendo las necesidades de quienes los confeccionaron.

\section{Agradecimientos}

Quiero agradecer a Alejandra Matarrese por su apoyo al momento del análisis de los materiales, a Luciano Prates por su revisión crítica del manuscrito y a Emiliano Mange por sus comentarios en la presentación del CAELA y su ayuda en la confección de las figuras, y a los evaluadores anónimos por sus sugerencias y comentarios. Este trabajo fue realizado en el marco del proyecto "Continuidad y cambio en los patrones de explotación de recursos en el centro de Río Negro durante el Holoceno tardío" (PICT-2015-3645 dirigido por L. Prates), y de los proyectos de beca (EVC-CIN 2016 y 2017).

\section{Bibliografía}

Adams, J. L. (2002). Ground Stone Analysis. A technological approach. The University of Utah Press, Salt Lake City.

Álvarez Soncini, M. C. (2018). Tecnologías de piqueteamiento y pulimentación en la dinámica socio-económica de sociedades cazadoras-recolectoras (Tesis Doctoral). Facultad de Ciencias Naturales y Museo, Universidad Nacional de La Plata, Buenos Aires, Argentina.

Andreis, R.A. (1965). Petrografía y paleocorrientes de la formación Río Negro. Revista del Museo de La Plata. (N.S.) 36 (5): 245-310.

Aschero, C. (1975). Ensayo para una clasificación morfológica de artefactos líticos aplicada a estudios tipológicos comparativos. Buenos Aires, Argentina, Informe al CONICET, Ms.

Aschero, C. (1983). Ensayo para una clasificación morfológica de artefactos líticos aplicada a estudios tipológicos comparativos. Revisión. Buenos Aires, Argentina, Informe al CONICET, Ms.

Babot, P. (2004). Tecnología y utilización de artefactos de molienda en el noroeste prehispánico (Tesis Doctoral). Facultad de Ciencias Naturales e Instituto Miguel Lillo, Universidad Nacional de Tucumán, Tucumán, Argentina.

Babot, P. (2006). El papel de la molienda en la transición hacia la producción agropastoril: Un análisis desde la Puna Meridional argentina. Estudios atacameños, (32), 75-92.

Babot, P. (2011). Cazadores-recolectores de los andes centrosur y procesamiento vegetal: Una discusión desde la puna meridional argentina (ca. 7.000-3.200 años AP). Chungará (Arica), 43(Especial), 413-432. 
Binford, L. R. (1979). Organization and formation processes: looking at curated technologies. Journal of Anthropological Research, 35 (3), 225-273.

Bonomo, M. y Prates, L. (2014). La explotación de depósitos secundarios de rodados en el curso medio del río Negro y el litoral marítimo pampeano. Artefactos líticos, movilidad y funcionalidad de sitios en Sudamérica. Problemas y perspectivas, 77-92.

Casamiquela, R. M. (1999). Proyecto etnobotánica de la Patagonia: Primer informe. En: Aspectos Técnicos, Culturales, Políticos y Legales de la Bioprospección en Argentina, 91-134.

De Ferrariis, C. I. (1966). Estudio estratigráfico de la Formación Río Negro de la Provincia de Buenos Aires. Sus relaciones con la Región Nordpatagónica. Anales de la Comisión de Investigaciones Científicas VII, 85- 165.

Di Prado, V. (2013). Del interior a la superficie: análisis de las pastas y las representaciones decorativas de la alfarería de loma de los muertos (este de Norpatagonia). Magallania (Punta Arenas), 41(2), 197-214.

Escosteguy, L., Etcheverría, M.P., Folguera, A., Franchi, M., Faroux, A.J. y Getin, P.R. (2011). Hoja Geológica 3966- IV, Choele Choel. Provincia de Río Negro. Instituto de Geología y Recursos Minerales, SEGEMAR. Boletín 398, pp. 38. Buenos Aires

González Díaz E. F. y Malagnino, E. C. (1984). Geomorfología de la provincia de Río Negro. En: Geología y Recursos Naturales de la Provincia de Río Negro: relatorio del IX congreso geológico argentino, 347-364. Asociación geológica argentina, San Carlos de Bariloche, Río Negro.

Heider, G. y López, L. (2016). El consumo de recursos vegetales silvestres en grupos cazadores recolectores del norte de pampa seca (San Luis y Córdoba, Argentina). Mundo de Antes, 10, 00.

Lema, V., Della Negra, C. y Bernal, V. (2012). Explotación de recursos vegetales silvestres y domesticados en Neuquén: implicancias del hallazgo de restos de maíz y algarrobo en artefactos de molienda del Holoceno tardío. Magallania (Punta Arenas), 40 (1), 229-249.

Luchsinger, H. (2006). The late Quaternary landscape history of the middle rio Negro valley, Northern Patagonia, Argentina: Its impact on preservation of the archaeological record and influence on Late Holocene human settlement patterns (Tesis doctoral), A\&M Texas University, College Station.

Mange, E. (2019). Investigaciones arqueológicas en la margen sur del valle medio-superior del río Negro (pcia. de Río Negro) (Tesis Doctoral). Facultad de Ciencias Naturales y Museo, Universidad Nacional de La Plata, Buenos Aires, Argentina.

Mange, E., Ramos van Raap, A., y Leon, D. C. (2013). La arqueofauna del sitio Loma de los Muertos (departamento de
General Conesa, Río Negro). Intersecciones en Antropología, 14 (2), 301-314.

Matarrese, A. (2015). Tecnología lítica entre los cazadoresrecolectores pampeanos: los artefactos formatizados por picado y abrasión y modificados por uso en el área Interserrana Bonaerense (Tesis Doctoral). Facultad de Ciencias Naturales y Museo, Universidad Nacional de La Plata, Buenos Aires, Argentina.

Nelson, M. (1991). The study of technological organization. In: Archaeological method and theory, 3, 57-100.

Prates, L. (2008). Los indígenas del río Negro. Un enfoque arqueológico. Colecciones Tesis doctorales de la Sociedad Argentina de Antropología.

Prates, L. (2009). El uso de los recursos por los cazadoresrecolectores posthispánicos de Patagonia continental y su importancia arqueológica. Relaciones de la Sociedad Argentina de Antropología, 34, 201-229.

Prates, L. (2014). Crossing the boundary between humans and animals: the extinct fox Dusicyon avus from a hunter-gatherer mortuary context in Patagonia (Argentina). Antiquity, 88 (342), 1201-1212.

Prates, L., Di Prado, V., Mange, E. y Serna, A. (2010a). Sitio Loma de los Muertos: Múltiples ocupaciones sobre un médano del Este de Norpatagonia (Argentina). Magallania (Punta Arenas), 38 (1), 165-181.

Prates, L., Flensborg, G. A., y Bayala, P. (2010b). Caracterización de los entierros humanos del sitio Loma de los Muertos (valle medio del río Negro, Argentina). Magallania (Punta Arenas), 38(1), 149-164.

Saghessi, D. y Mange, E. (2017). Artefactos picados y/o abradidos procedentes de sitios arqueológicos y colecciones del valle medio del río Negro (Río Negro, Argentina). Revista del Museo La Plata, 2 (1), Suplemento Resúmenes: 12R.

Suriano, J.M., Ferro, G. y Dalponte, M. (1999). Convenio Dirección de Minería de Río Negro-SEGEMAR: Geología y Recursos Minerales de la Hoja 3966-IV, Choele Choel y del Sector Rionegrino de las Hojas 3963-III, Colonia Juliá; 4163-I-III, General Conesa y 4163-II-IV, Viedma. Instituto de Geología y Recursos Minerales, Viedma.

Vecchi, R. (2011). Bolas de boleadora en los grupos cazadoresrecolectores de la pampa bonaerense (Tesis doctoral). Facultad de Filosofía y Letras, Universidad Nacional de Buenos Aires, Buenos Aires, Argentina.

Vecchi, R. J. y González, M. I. (2018). Artefactos formatizados por picado, abrasión, pulido y modificados por uso en la depresión del Río Salado, Provincia de Buenos Aires, Argentina. Revista del Museo de Antropología, 11 (2), 85-100. 